\title{
Author Correction: Oppression or Opportunity? Sexual Strategies and the Perception of Sexual Advances
}

\author{
Lisa Klümper $^{1}$ - Sascha Schwarz ${ }^{1}$ \\ Published online: 18 December 2019 \\ (C) Springer Nature Switzerland AG 2019
}

\section{Author Correction: Evolutionary Psychological Science} https://doi.org/10.1007/s40806-019-00215-y

The original version of this article contained a mistake. In Table 2, there are two values that are displayed wrong. The correct Table 2 is shown here.

Table 2 Descriptive statistics of SOI-R scores, SOI-R facets and mean perceived harmfulness of the scenarios as a function of sex in study 1 and study 2

\begin{tabular}{|c|c|c|c|c|c|c|}
\hline & \multicolumn{2}{|l|}{ Study 1} & \multicolumn{4}{|l|}{ Study 2} \\
\hline & \multicolumn{2}{|c|}{ Attractiveness } & \multicolumn{2}{|c|}{ Physical appearance } & \multicolumn{2}{|l|}{ Status } \\
\hline & $\begin{array}{l}\text { Men } \\
(n=105) \\
M(S D)\end{array}$ & $\begin{array}{l}\text { Women } \\
(n=411) \\
M(S D)\end{array}$ & $\begin{array}{l}\text { Men } \\
(n=250) \\
M(S D)\end{array}$ & $\begin{array}{l}\text { Women } \\
(n=251) \\
M(S D)\end{array}$ & $\begin{array}{l}\text { Men } \\
(n=228) \\
M(S D)\end{array}$ & $\begin{array}{l}\text { Women } \\
(n=271) \\
M(S D)\end{array}$ \\
\hline SOI-R & $3.19(0.92)$ & $2.50(0.77)$ & $3.06(0.82)$ & $2.52(0.83)$ & $2.99(0.79)$ & $2.46(0.80)$ \\
\hline SOI-R attitudes & $3.76(1.14)$ & $3.05(1.09)$ & $3.61(1.08)$ & $3.25(1.22)$ & $3.63(1.07)$ & $3.14(1.24)$ \\
\hline SOI-R behavior & $2.65(1.16)$ & $2.22(0.90)$ & $2.41(1.14)$ & $2.13(0.94)$ & $2.35(1.07)$ & $2.09(0.90)$ \\
\hline SOI-R desire & $3.17(1.08)$ & $2.23(0.89)$ & $3.17(1.04)$ & $2.18(0.91)$ & $2.99(0.99)$ & $2.14(0.86)$ \\
\hline Harmfulness of scenarios & $4.14(1.37)$ & $5.04(0.81)$ & $3.73(1.21)$ & $5.16(0.82)$ & $3.57(1.15)$ & $5.21(0.70)$ \\
\hline
\end{tabular}

SOI-R and its facets were measured on 5-point scales. Perceived harmfulness across all three scenarios varies from 1: "not at all" to 6: "very"

The online version of the original article can be found at https://doi.org/ 10.1007/s40806-019-00215-y

Lisa Klümper

lisa.kluemper@uni-wuppertal.de

1 School of Human and Social Sciences, Social and Personality Psychology, Bergische Universität Wuppertal, Gaußstr. 20, 42119 Wuppertal, Germany 